DESY 10-184

DO-TH 10/20

ZU-TH-17/10

SFB-CPP/10-118

November 2010

\title{
NNLO Benchmarks for Gauge and Higgs Boson Production at TeV Hadron Colliders
}

\author{
S. Alekhin ${ }^{a, b 1}$, J. Blümlein ${ }^{a}$, P. Jimenez-Delgado ${ }^{c}$, S. Moch $^{a}$, and E. Reya ${ }^{d}$ \\ a Deutsches Elektronen-Synchrotron, DESY, \\ Platanenallee 6, D-15738 Zeuthen, Germany \\ ${ }^{b}$ Institute for High Energy Physics \\ 142281 Protvino, Moscow region, Russia \\ c Institut für Theoretische Physik, Universität Zürich, \\ Winterthurer Str. 190, CH-8057 Zürich, Switzerland \\ ${ }^{d}$ Technische Universität Dortmund, Institut für Physik, \\ D-44221 Dortmund, Germany
}

\begin{abstract}
The inclusive production cross sections for $W^{+}, W^{-}$and $Z^{0}$-bosons form important benchmarks for the physics at hadron colliders. We perform a detailed comparison of the predictions for these standard candles based on recent next-to-next-to-leading order (NNLO) parton parameterizations and new analyses including the combined HERA data, compare to all available experimental results, and discuss the predictions for present and upcoming RHIC, SPS, Tevatron and LHC energies. The rates for gauge boson production at the LHC can be rather confidently predicted with an accuracy of better than about $10 \%$ at NNLO. We also present detailed NNLO predictions for the Higgs boson production cross sections for Tevatron and LHC energies $(1.96,7,8,14 \mathrm{TeV})$, and propose a possible method to monitor the gluon distribution experimentally in the kinematic region close to the mass range expected for the Higgs boson. The production cross sections of the Higgs boson at the LHC are presently predicted with an accuracy of about $10-17 \%$. The inclusion of the NNLO contributions is mandatory for achieving such accuracies since the total uncertainties are substantially larger at NLO.
\end{abstract}

\footnotetext{
${ }^{1}$ Helmholtz Alliance Fellow
} 
1 Introduction. The inclusive production cross sections of the weak gauge bosons $W^{ \pm}, Z^{0}$ and of the Higgs particle $H^{0}$ form important reference points for the physics at hadron colliders. Within QCD the corresponding cross sections have been calculated to NNLO [1,2]. ${ }^{2}$ This level of accuracy is necessary to control the renormalization and factorization scale uncertainties of the parton distribution functions (pdfs), which are still of significant size at next-to-leading order (NLO). The rapidly growing luminosity at the Large Hadron Collider, LHC, allows a precise measurement of these quantities [6,7], which reaches the accuracy of the NNLO predictions, based on the world deep-inelastic, Drell-Yan and di-muon data, and part of the Tevatron data [8-13]. These analyses also require a correct treatment of the heavy flavor contributions, cf. [14], which yield sizeable effects.

In this note we provide NNLO predictions for the inclusive weak boson and Standard Model Higgs-boson production cross sections, and compare to the available experimental data $[6,7,15-$ 20]. For present and upcoming experimental analyses we also provide detailed reference tables, and comment on the well-known NLO predictions, cf. [21]. These are, however, far less precise due to their inherent large renormalization and factorization scale uncertainties [22, 23].

The effect of the new combined HERA data on the inclusive NNLO $W^{ \pm}, Z^{0}$ and $H^{0}$ boson cross sections [24] is shown by comparing the results for the recent ABM10 [8] and ABKM09 [9] distributions. It leads to significant shifts of the total rate of about $1 \sigma$ in the pdf-error for a wide range of collider energies and processes. We consider all collider energies having been probed so far, compare to all measurements, and give predictions for the high energy options at the LHC. In this way a wide kinematic region in Bjorken $x$ is probed for the corresponding parton luminosities which may help to delineate remaining differences in the current NNLO pdfs and to devise a way of potential further improvements.

$2 \mathrm{~W}^{ \pm}$and $Z^{0}$ Boson Production. The inclusive $W^{ \pm}$and $Z^{0}$ boson production cross sections in $p \bar{p}$ and $p p$ scattering are known to 2nd order (NNLO) in the strong coupling constant [1], supplemented by the 1st order (NLO) electroweak corrections, cf. [3]. In the following comparison we will concentrate on the QCD corrections only. The electroweak parameters are calculated choosing the scheme based on $\left(G_{F}, M_{W}, M_{Z}\right)$ [25] with $G_{F}=1.16637 \times 10^{-5} \mathrm{GeV}^{-2}, M_{W}=$ $80.399 \pm 0.023 \mathrm{GeV}, M_{Z}=91.1876 \pm 0.0021 \mathrm{GeV}$, and the weak mixing angle as dependent quantity, with

$$
\hat{s}_{Z}^{2}=1-\frac{M_{W}^{2}}{\hat{\rho} M_{Z}^{2}}=0.2307 \pm 0.0005,
$$

and $\hat{\rho}=1.01047 \pm 0.00015$. Furthermore, the width of the $W^{ \pm}$and $Z^{0}$ bosons are $\Gamma\left(W^{ \pm}\right)=$ $2.085 \pm 0.042 \mathrm{GeV}, \Gamma\left(Z^{0}\right)=2.4952 \pm 0.0023 \mathrm{GeV}$, and $\sin ^{2} \theta_{c}=0.051$, with $\theta_{c}$ the Cabibbo angle. We compute the inclusive production cross sections at various collider energies for the recent NNLO parton distributions, ABM10 [8], ABKM09 [9], JR [10], MSTW08 [11], and HERAPDF [12]. In the latter case we refer to the fit where a value of $\alpha_{s}\left(M_{Z}^{2}\right)=0.1145$ has been assumed at NNLO, which resulted in the lowest value for $\chi_{\min }^{2}$ among other choices. ${ }^{3}$

In Table 1 we summarize the cross sections for the different $p \bar{p}$ collision energies at the SPS and the Tevatron at NNLO for the distributions [8-12]. For one of the parameterizations, JR, we compare also to the NLO QCD corrections. The relative differences between the NLO and NNLO corrections are of comparable size for all other pdf sets. We also list the corresponding NNLO values of $\alpha_{s}\left(M_{Z}^{2}\right)$ and their errors as determined, or partly being assumed, in the various

\footnotetext{
${ }^{2}$ Electro-weak corrections were calculated in [3-5].

${ }^{3}$ For the time being the NNLO HERAPDF parameterization yields only central values, unlike the NLO distributions, where also errors are provided, cf. [12].
} 
analyses, which in most cases turn out to be similar. We note that the corresponding $\alpha_{s}$ values at NLO, despite differences in the central values, necessarily all agree within the rather large theory errors due to factorization and renormalization scale uncertainties of at least \pm 0.005 , cf. [22,23]. This also applies to other NLO analyses [26,27].

\begin{tabular}{|c|c|c|c|c|c|}
\hline$\sqrt{s}(\mathrm{TeV})$ & & 0.546 & 0.630 & 1.8 & 1.96 \\
\hline ABM10 [8] & $W^{ \pm}$ & $5.632 \pm 0.092$ & $7.045 \pm 0.111$ & $24.441 \pm 0.235$ & $26.740 \pm 0.259$ \\
$\alpha_{s}=0.1147 \pm 0.0012$ & $Z^{0}$ & $1.761 \pm 0.022$ & $2.187 \pm 0.028$ & $7.181 \pm 0.068$ & $7.846 \pm 0.075$ \\
\hline ABKM09 [9] & $W^{ \pm}$ & $5.804 \pm 0.075$ & $7.222 \pm 0.091$ & $23.88 \pm 0.243$ & $26.09 \pm 0.265$ \\
$\alpha_{s}=0.1135 \pm 0.0014$ & $Z^{0}$ & $1.806 \pm 0.020$ & $2.234 \pm 0.024$ & $7.056 \pm 0.068$ & $7.691 \pm 0.075$ \\
\hline JR [10] & $W^{ \pm}$ & $5.983 \pm 0.148$ & $7.346 \pm 0.159$ & $23.069 \pm 0.238$ & $25.157 \pm 0.251$ \\
$\alpha_{s}=0.1124 \pm 0.0020$ & & $(5.358 \pm 0.152)$ & $(6.637 \pm 0.167)$ & $(22.121 \pm 0.274)$ & $(24.181 \pm 0.296)$ \\
& $Z^{0}$ & $1.837 \pm 0.029$ & $2.268 \pm 0.034$ & $6.975 \pm 0.071$ & $7.586 \pm 0.076$ \\
\hline MSTW08 [11] & $W^{ \pm}$ & $5.469 \pm 0.151$ & $6.802 \pm 0.176$ & $23.14 \pm 0.394$ & $25.35 \pm 0.422$ \\
$\alpha_{s}=0.1171 \pm 0.014$ & $Z^{0}$ & $1.654 \pm 0.047$ & $2.056 \pm 0.056$ & $6.773 \pm 0.126$ & $7.406 \pm 0.134$ \\
\hline HERAPDF [12] & $W^{ \pm}$ & 6.121 & 7.519 & 24.51 & 26.80 \\
$\alpha_{s}=0.1145$ & $Z^{0}$ & 1.853 & 2.296 & 7.319 & 7.978 \\
\hline
\end{tabular}

Table 1: NNLO predictions for the production cross sections $\sigma(p \bar{p} \rightarrow V+X)[n b]$, with $V=W^{ \pm}, Z^{0}$. The abbreviation $W^{ \pm}$refers to the sum $W^{+}+W^{-}$. Notice that for $p \bar{p}$ collisions the $W^{+}$and $W^{-}$ cross sections are equal. The errors refer to the $\pm 1 \sigma$ pdf uncertainties. The NNLO values of $\alpha_{s}$ refer to $\alpha_{s}=\alpha_{s}\left(M_{Z}^{2}\right)$. To allow for a comparison with the corrections up to NLO the corresponding cross sections for the JR distributions are also listed as an example in parentheses [28].

The NNLO corrections furthermore enhance the cross sections at all center-of-mass (cms) energies both for $W^{ \pm}$and $Z^{0}$ boson production. The importance of full NNLO analyses becomes evident when comparing the NLO and NNLO predictions in Tables 1 and 2. At the Tevatron $(1.96 \mathrm{TeV})$, for example, $\Delta \sigma^{W^{ \pm}} \equiv \sigma_{\mathrm{NNLO}}^{W^{ \pm}}-\sigma_{\mathrm{NLO}}^{W^{ \pm}}=+0.930,+0.976$ and $+0.825 n b$ for ABM10, JR and MSTW08, respectively. Similarly for LHC (7 TeV) one obtains $\Delta \sigma^{W^{ \pm}}=+1.05,+2.36$ and $+2.46 n b$ for ABM10, JR and MSTW08, respectively. This corresponds at the Tevatron to a difference of more than $3 \sigma$ w.r.t. the pdf-errors, and to more than $1 \sigma$ at LHC. Furthermore, at NNLO the parton distributions are much more stable against renormalization and factorization scale uncertainties than at NLO : the scale variations of the full NNLO predictions in Table 1 amount to less than $0.5 \%$ (i.e., are about half as large as the stated $1 \sigma_{\text {pdf }}$ uncertainties) which is about four times smaller than at NLO [10,28]; at LHC energies the scale uncertainties of the NNLO predictions in Table 2 amount to less than $2 \%$ of the total predicted rates which is about half as large as the stated $1 \sigma_{\text {pdf }}$ uncertainties and the scale uncertainties at NLO $[10,28]$.

Thus far, the combined H1 and ZEUS data [24] have been taken into account in the ABM10 [8] and HERAPDF [12] analyses only, and their relative effect can be seen by comparing the numbers for the ABM10 and ABKM09 distributions at NNLO. While for the lower collider energies 2$3.5 \%$ lower cross sections are obtained, about $2.5 \%$ larger cross sections result for the Tevatron energies of 1.8 and $1.96 \mathrm{TeV}$. With respect to the current pdf-errors this effect amounts to $2 \sigma$. 


\begin{tabular}{|c|c|c|c|c|c|}
\hline$\sqrt{s}(\mathrm{TeV})$ & & 0.5 & 7 & 10 & 14 \\
\hline ABM10 [8] & $W^{+}$ & $1.236 \pm 0.057$ & $59.86 \pm 0.838$ & $85.58 \pm 1.267$ & $118.4 \pm 1.891$ \\
& $W^{-}$ & $0.363 \pm 0.092$ & $40.28 \pm 0.535$ & $60.28 \pm 0.852$ & $86.58 \pm 1.331$ \\
& $W^{ \pm}$ & $1.600 \pm 0.070$ & $100.1 \pm 1.315$ & $145.9 \pm 2.065$ & $205.0 \pm 3.186$ \\
& $Z^{0}$ & $0.305 \pm 0.015$ & $29.01 \pm 0.391$ & $42.77 \pm 0.633$ & $60.69 \pm 0.963$ \\
\hline ABKM09 [9] & $W^{+}$ & $1.160 \pm 0.046$ & $58.86 \pm 0.903$ & $85.14 \pm 1.427$ & $119.4 \pm 2.072$ \\
& $W^{-}$ & $0.348 \pm 0.014$ & $39.43 \pm 0.614$ & $59.56 \pm 0.993$ & $86.53 \pm 1.525$ \\
& $W^{ \pm}$ & $1.509 \pm 0.058$ & $98.27 \pm 1.527$ & $144.7 \pm 2.436$ & $205.9 \pm 3.658$ \\
& $Z^{0}$ & $0.287 \pm 0.012$ & $28.42 \pm 0.457$ & $42.28 \pm 0.743$ & $60.70 \pm 0.115$ \\
\hline JR $[10]$ & $W^{+}$ & $1.138 \pm 0.061$ & $54.57 \pm 1.10$ & $78.43 \pm 1.98$ & $109.31 \pm 3.13$ \\
& & $(1.245 \pm 0.065)$ & $(52.96 \pm 0.99)$ & $(76.60 \pm 1.74)$ & $(107.58 \pm 2.95)$ \\
& $W^{-}$ & $0.387 \pm 0.028$ & $37.15 \pm 0.79$ & $55.54 \pm 1.44$ & $80.02 \pm 2.31$ \\
& & $(0.427 \pm 0.030)$ & $(36.39 \pm 0.72)$ & $(54.67 \pm 1.26)$ & $(79.16 \pm 2.12)$ \\
& $W^{ \pm}$ & $1.525 \pm 0.052$ & $91.72 \pm 1.82$ & $133.99 \pm 3.35$ & $189.29 \pm 5.41$ \\
& & $(1.672 \pm 0.053)$ & $(89.36 \pm 1.57)$ & $(131.23 \pm 2.87)$ & $(186.74 \pm 4.95)$ \\
& $Z^{0}$ & $0.300 \pm 0.011$ & $27.24 \pm 0.50$ & $40.39 \pm 0.95$ & $57.85 \pm 1.56$ \\
& & $(0.336 \pm 0.012)$ & $(26.57 \pm 0.43)$ & $(39.57 \pm 0.81)$ & $(57.00 \pm 1.42)$ \\
\hline HERAPDF [12] & $W^{+}$ & 1.219 & 59.37 & 85.37 & 119.0 \\
& $W^{-}$ & 0.414 & 40.82 & 61.06 & 87.94 \\
& $W^{ \pm}$ & 1.633 & 100.2 & 146.4 & 206.9 \\
& $Z^{0}$ & 0.322 & 29.08 & 42.95 & 61.22 \\
\hline MSTW08 [11,13] & $W^{+}$ & $1.221 \pm 0.0421$ & $56.80 \pm 0.971$ & $81.83 \pm 1.405$ & $114.0 \pm 1.945$ \\
& $W^{-}$ & $0.416 \pm 0.017$ & $39.63 \pm 0.678$ & $59.45 \pm 1.008$ & $85.63 \pm 1.484$ \\
& $1.637 \pm 0.052$ & $96.41 \pm 1.607$ & $141.3 \pm 2.372$ & $199.6 \pm 3.379$ \\
& $0.319 \pm 0.011$ & $27.89 \pm 0.481$ & $41.34 \pm 0.705$ & $58.99 \pm 1.012$ \\
\hline
\end{tabular}

Table 2: NNLO predictions for the production cross sections $\sigma(p p \rightarrow V+X)[n b]$, with $V=W^{ \pm}, Z^{0}$. The abbreviation $W^{ \pm}$denotes the sum $W^{+}+W^{-}$. The errors refer to the $\pm 1 \sigma$ pdf uncertainties. To allow for a comparison with the corrections up to NLO we also listed the corresponding cross sections for the JR distributions as an example in parentheses [28].

For illustration we compare in Figure 1(a) the different predictions [8, 10-12] with each other and with the measured cross sections $[15-20]$ at fixed energies in the range $\sqrt{S}=0.5-1.96$ $\mathrm{TeV}$, covering also the $p p$ cross sections at RHIC, cf. Table 2. A detailed summary of the various measurements is given in the Appendix. As can also be seen in Table 1 the results for $Z^{0}$ production predicted by the different parameterizations agree somewhat better than in the case of $W^{ \pm}$production. The experimental errors at lower energies $\sqrt{S}<0.63 \mathrm{TeV}$ are rather large and the current NNLO predictions agree with experiment. For $\sqrt{S}=0.63 \mathrm{TeV}$ all NNLO analyses predict cross sections which are at the upper end of the measurements. At Tevatron energies the ABM10 and HERAPDF distributions yield about $5-7 \%$ larger $W^{ \pm}$cross sections than those of JR and MSTW08. Moving to higher energies the JR distributions result in lower cross sections as compared to the ABM10 and HERAPDF distributions, which yield 
larger values at lower energies as well. Yet all predictions are in agreement with the current experimental results at the level of $1.5 \sigma$.
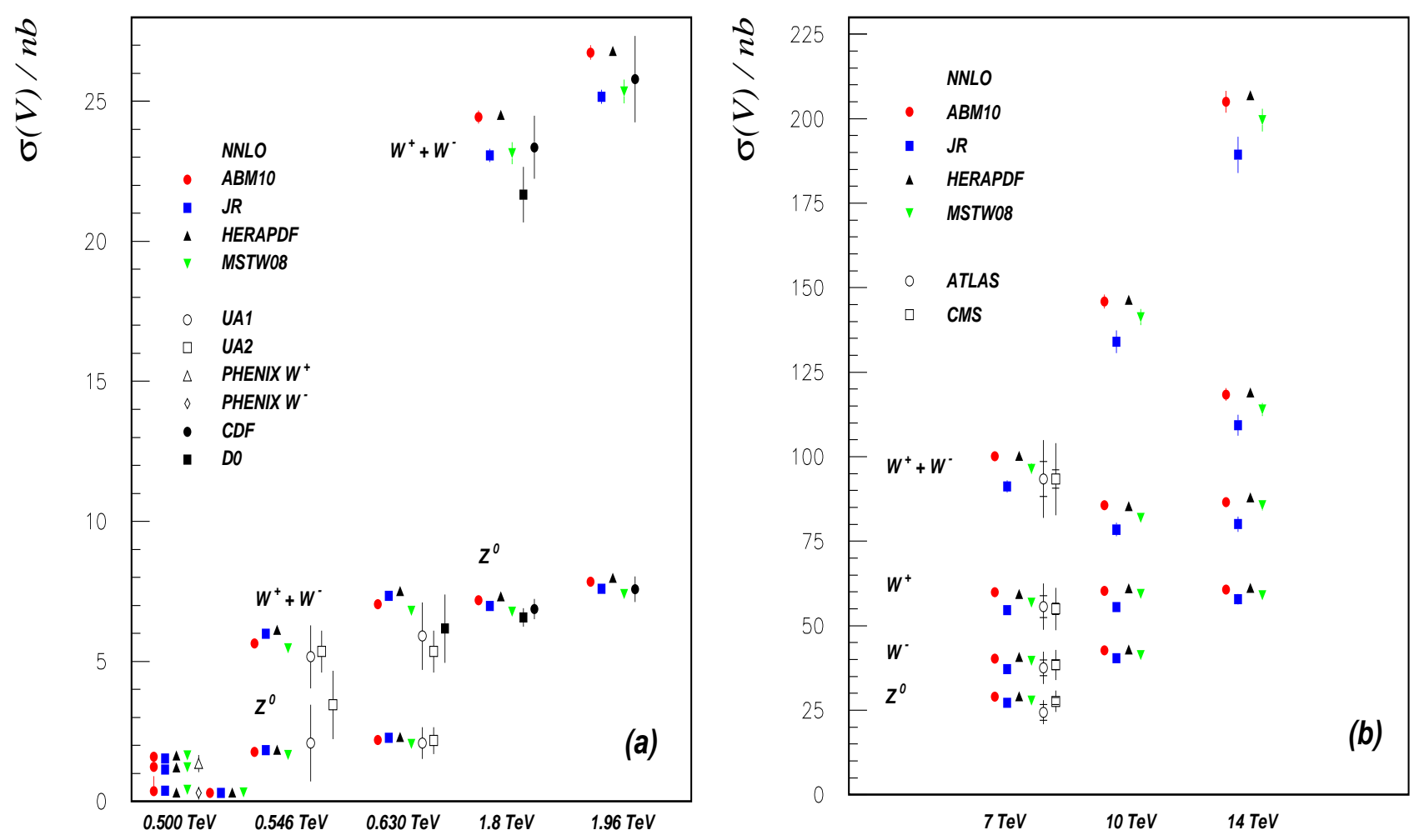

Figure 1: Comparison of different NNLO predictions for the inclusive $W^{+}, W^{-}, W^{ \pm}$, and $Z^{0}$ boson production cross sections in $p \bar{p}$ annihilation and $p p$ scattering $(\sqrt{S}=0.5 \mathrm{TeV})$ based on the pdfs of recent NNLO analyses [8-13] and the corresponding experimental data [6,7,15-20]. Left panel (a): the lower energy region corresponds to $p \bar{p}$ collisions, except at $0.5 \mathrm{TeV}$, which refers to $p p$ scattering. For the latter case the predictions refer to (from above) $W^{+}+W^{-}, W^{+}, W^{-}$and the ones for $Z^{0}$ are given to the right of the ones for $W^{-}$. Right panel (b): LHC energies ( $p p$ collisions); the inner error bars refer to $\left(\sigma_{\text {stat }}^{2}+\sigma_{\text {syst }}^{2}\right)^{1 / 2}$ and the total error is obtained by adding the luminosity error in quadrature.

In Table 2 we list the NNLO predictions for the $p p$ production cross sections for $W^{+}, W^{-}$ bosons, their sum, as well as those for $Z^{0}$ bosons at RHIC and for different present or planned collider energies at the LHC. As before we supplement the NLO prediction for one set of distributions (JR) to allow for comparisons as an example. For the LHC energies the NNLO corrections lead to an enhancement of the cross sections w.r.t. NLO, while at RHIC energies the NNLO cross sections become smaller. At LHC energies the pattern of relative differences of the various predictions [8-13] both for $W^{ \pm}$and $Z^{0}$ boson production pertains over the whole energy range, see also Figure 1(b). The cross sections grow empirically nearly linearly with $\sqrt{S}$. The impact of the combined HERA data is less than at lower collider energies and leads in most cases only to a very slight enhancement of the cross sections, as a comparison of the values in Table 2 resulting from the ABM10 and ABKM09 distributions shows. The largest scattering cross sections are obtained for the ABM10 and HERAPDF distributions, followed by slightly lower values for MSTW08, and even somewhat lower cross sections are obtained for the JR distributions, with differences of up to $9 \%$. Comparing to the first experimental measurements by CMS [6] and ATLAS [7], all current predictions are compatible within the present experimental errors for all channels.

Due to the reduced scale uncertainty at NNLO and the slightly different NNLO estimates of the various groups, cf. Figures $1(\mathrm{a})$ and (b), and Tables 1 and 2, we conclude that the 
rates for gauge boson production at LHC energies can be rather confidently predicted with an accuracy better than about $9 \%$. These differences are due to the different light sea distributions $(\bar{u}, \bar{d}, \bar{s})$ obtained in the various analyses and require more detailed theoretical and experimental investigations in the future.

3 Higgs Boson Production. The inclusive partonic production cross section ${ }^{4}$ of the Higgs boson of the Standard Model $\sigma\left(p p \rightarrow H^{0}+X\right)$ [2] depends on the strong coupling constant $\propto \alpha_{s}^{2}$ and a large part of the cross section exhibits a quadratic dependence of the gluon density. As has been shown in Table 1, the $\alpha_{s}\left(M_{Z}^{2}\right)$ values determined, resp. assumed [12], are still different. Despite having reached an impressive accuracy of $O(1 \%)$ in individual analyses of the world deep-inelastic data and related collider data, $[8-10,29,31]^{5}$, and for a large number of other high energy processes and decay widths, cf. [33], an overall agreement has not yet been reached. As well-known, the gluon density and the value of $\alpha_{s}\left(M_{Z}^{2}\right)$ are anticorrelated, see e.g. the tables of

\begin{tabular}{|c|c|c|c|c|c|}
\hline$M_{H}(\mathrm{GeV})$ & ABM10 $[8]$ & ABKM09 $[9]$ & JR $[10]$ & MSTW08 $[11]$ & HERAPDF $[12]$ \\
\hline 100 & $1.438 \pm 0.066$ & $1.380 \pm 0.076$ & $1.593 \pm 0.091$ & $1.682 \pm 0.046$ & 1.417 \\
110 & $1.051 \pm 0.052$ & $1.022 \pm 0.061$ & $1.209 \pm 0.078$ & $1.265 \pm 0.038$ & 1.055 \\
115 & $0.904 \pm 0.047$ & $0.885 \pm 0.055$ & $1.060 \pm 0.072$ & $1.104 \pm 0.034$ & 0.917 \\
120 & $0.781 \pm 0.042$ & $0.770 \pm 0.050$ & $0.933 \pm 0.067$ & $0.968 \pm 0.031$ & 0.800 \\
125 & $0.677 \pm 0.038$ & $0.672 \pm 0.045$ & $0.823 \pm 0.062$ & $0.851 \pm 0.029$ & 0.700 \\
130 & $0.588 \pm 0.034$ & $0.589 \pm 0.041$ & $0.729 \pm 0.058$ & $0.752 \pm 0.026$ & 0.615 \\
135 & $0.513 \pm 0.031$ & $0.518 \pm 0.037$ & $0.647 \pm 0.054$ & $0.666 \pm 0.024$ & 0.541 \\
140 & $0.449 \pm 0.028$ & $0.456 \pm 0.034$ & $0.576 \pm 0.050$ & $0.591 \pm 0.022$ & 0.479 \\
145 & $0.394 \pm 0.025$ & $0.403 \pm 0.031$ & $0.514 \pm 0.047$ & $0.527 \pm 0.020$ & 0.424 \\
150 & $0.347 \pm 0.023$ & $0.358 \pm 0.028$ & $0.461 \pm 0.044$ & $0.471 \pm 0.018$ & 0.377 \\
155 & $0.306 \pm 0.020$ & $0.318 \pm 0.026$ & $0.413 \pm 0.041$ & $0.421 \pm 0.017$ & 0.336 \\
160 & $0.271 \pm 0.019$ & $0.283 \pm 0.024$ & $0.371 \pm 0.039$ & $0.378 \pm 0.016$ & 0.300 \\
165 & $0.240 \pm 0.017$ & $0.253 \pm 0.022$ & $0.335 \pm 0.036$ & $0.341 \pm 0.014$ & 0.269 \\
170 & $0.213 \pm 0.015$ & $0.226 \pm 0.020$ & $0.302 \pm 0.034$ & $0.307 \pm 0.013$ & 0.241 \\
175 & $0.190 \pm 0.014$ & $0.203 \pm 0.019$ & $0.274 \pm 0.032$ & $0.278 \pm 0.012$ & 0.217 \\
180 & $0.169 \pm 0.013$ & $0.182 \pm 0.017$ & $0.248 \pm 0.030$ & $0.251 \pm 0.012$ & 0.195 \\
185 & $0.151 \pm 0.012$ & $0.164 \pm 0.016$ & $0.225 \pm 0.028$ & $0.228 \pm 0.011$ & 0.176 \\
190 & $0.136 \pm 0.011$ & $0.148 \pm 0.015$ & $0.205 \pm 0.027$ & $0.207 \pm 0.010$ & 0.159 \\
200 & $0.109 \pm 0.009$ & $0.121 \pm 0.013$ & $0.170 \pm 0.024$ & $0.172 \pm 0.009$ & 0.131 \\
\hline
\end{tabular}

Table 3: NNLO predictions for the production cross sections $\sigma\left(p \bar{p} \rightarrow H^{0}+X\right)[p b]$ at $\sqrt{S}=1.96 \mathrm{TeV}$. The errors refer to the $\pm 1 \sigma$ pdf uncertainties.

the correlation coefficients given in [9]. The values of $\alpha_{s}\left(M_{Z}^{2}\right)$ determined in global analyses to derive the parton distribution functions have all lower central values than the world average

\footnotetext{
${ }^{4}$ The vector boson fusion channel adds a correction of about $10 \%$, with an $O(2 \%)$ pdf uncertainty, cf. [30].

${ }^{5}$ For a compilation see Ref. [32].
} 

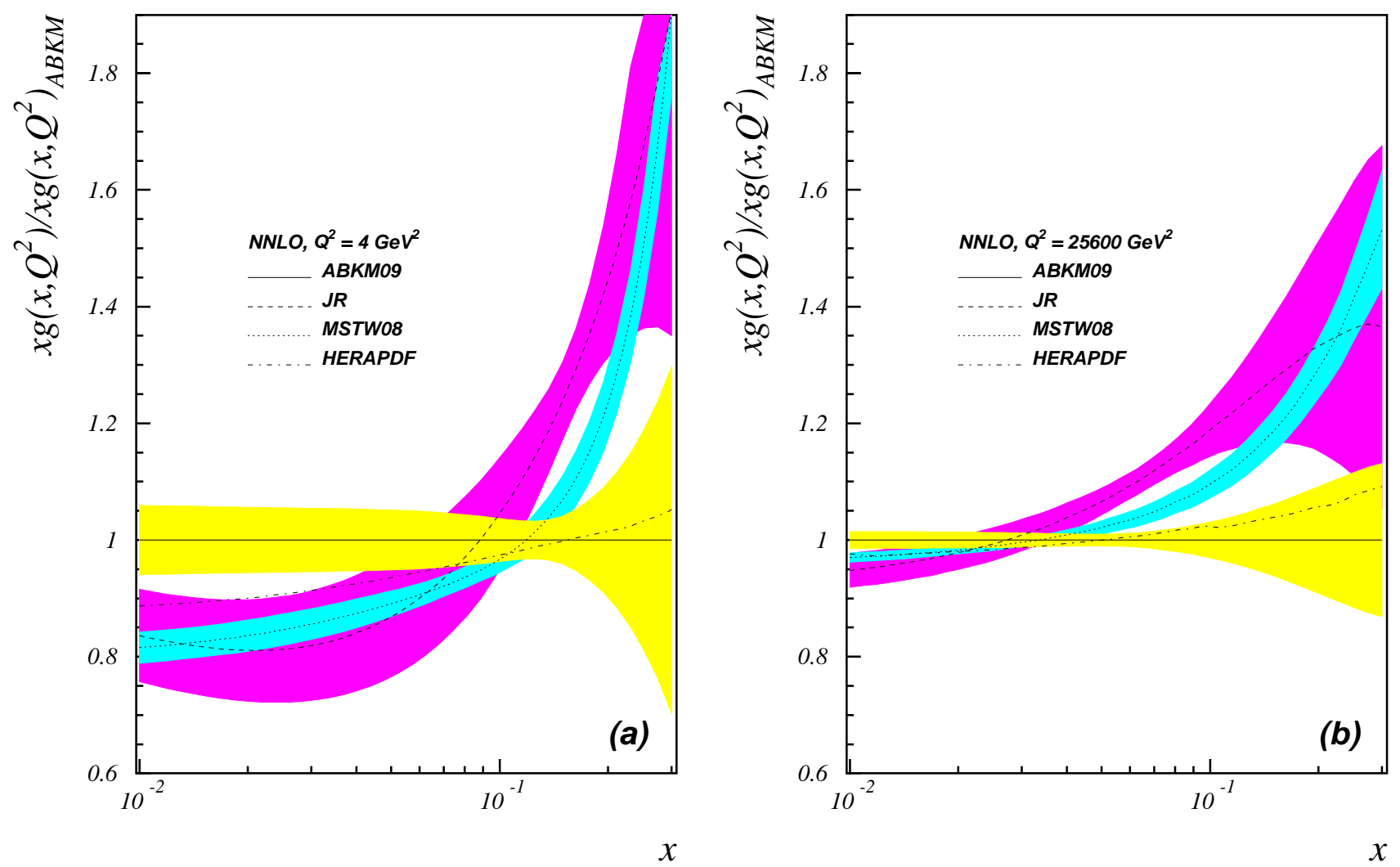

Figure 2: Comparison of the NNLO gluon distributions at $Q^{2}=4 \mathrm{GeV}^{2}$ and $Q^{2}=(160 \mathrm{GeV})^{2}$ for the ratios $x g\left(x, Q^{2}\right) / x g\left(x, Q^{2}\right)_{\text {ABKM }}$ for ABKM09 [9] (full line), JR [10] (dashed line), MSTW08 [11] (dotted line), and HERAPDF [12] (dash-dotted line, without error band).

in $[33]^{6}$, with a spread of $\Delta \alpha_{s}\left(M_{Z}^{2}\right)=0.0047$. If one extended the uncertainty referring to the average $\alpha_{s}\left(M_{Z}^{2}\right)$-value for the $\tau$-decay [33] one would obtain $\Delta \alpha_{s}\left(M_{Z}^{2}\right)=0.0073$. Both uncertainties imply

$$
\Delta \sigma\left(p \bar{p}(p) \rightarrow H^{0}+X\right) \sim\left(\frac{\Delta \alpha_{s}}{\alpha_{s}}\right)^{2}=8.4 \text { resp. } 13 \%
$$

Furthermore, there are also still significant differences in the gluon distributions of different analyses. The non-perturbative parton distributions at the initial scale $Q_{0}^{2}$ of the evolution are orthogonal in parameter space to $\Lambda_{\mathrm{QCD}}$, resp. $\alpha_{s}\left(M_{Z}^{2}\right)$, despite correlations. The evolution is therefore a direct mapping of the initial conditions and its strength is related to the value of $\alpha_{s}\left(M_{Z}^{2}\right)$. The larger this parameter is, the faster the evolution. In Figure 2 we compare the NNLO gluon distributions of ABMKM09, JR, HERAPDF, and MSTW08 at a scale $Q^{2}=4 \mathrm{GeV}^{2}$ and $Q^{2}=(160 \mathrm{GeV})^{2}$, a typical mass scale for current Higgs boson searches, in the relevant $x$-range. Average values of Bjorken $\langle x\rangle \sim 10^{-1},\langle x\rangle=\sqrt{x_{1} x_{2}}=M_{H} / \sqrt{S}$, in Figure 2(b) correspond to the production region at Tevatron, while those at $\langle x\rangle \sim 10^{-2}\left(3 \times 10^{-2}\right)$ are characteristic for the LHC at $\sqrt{S}=14(7) \mathrm{TeV}$. At $Q^{2}=4 \mathrm{GeV}^{2}$ the gluon distributions of JR and MSTW08 agree rather well in the region $x \in\left[10^{-2}, 10^{-1}\right]$, while ABM10 and HERAPDF yield $20 \%$ and $10 \%$ larger values at $x=10^{-2}$. All distributions get close for $x \sim 10^{-1}$ and for higher values the JR distributions becomes largest, followed by MSTW08 and HERAPDF. The evolution to $\mu^{2}=(160 \mathrm{GeV})^{2}$ diminishes the differences at $x \sim 10^{-2}$ overall to about $5 \%$. All distributions cross around $x \sim 3 \times 10^{-2}$, and at $x \sim 10^{-1}$ the gluon distributions by JR (MSTW08) take

\footnotetext{
${ }^{6}$ For other high energy measurements of $\alpha_{s}\left(M_{Z}^{2}\right)$ yielding lower values see e.g. [34].
} 
$\sim 20 \%$ (10\%) larger values than ABKM09, while the HERAPDF values are close to the latter. Note that JR obtains a $4 \%$ smaller value of $\alpha_{s}\left(M_{Z}^{2}\right)$ than MSTW08, and the value of $\alpha_{s}\left(M_{Z}^{2}\right)$ determined by ABKM is $3 \%$ smaller than that of MSTW08.

\begin{tabular}{|c|c|c|c|c|c|}
\hline$M_{H}(\mathrm{GeV})$ & ABM10 $[8]$ & ABKM09 $[9]$ & JR $[10]$ & MSTW08 $[11]$ & HERAPDF $[12]$ \\
\hline 100 & $22.82 \pm 0.53$ & $21.18 \pm 0.60$ & $20.48 \pm 0.70$ & $22.95 \pm 0.31$ & 20.90 \\
110 & $18.65 \pm 0.44$ & $17.30 \pm 0.49$ & $16.92 \pm 0.56$ & $18.84 \pm 0.26$ & 17.12 \\
115 & $16.95 \pm 0.40$ & $15.72 \pm 0.45$ & $15.46 \pm 0.50$ & $17.16 \pm 0.23$ & 15.58 \\
120 & $15.45 \pm 0.37$ & $14.34 \pm 0.41$ & $14.17 \pm 0.45$ & $15.69 \pm 0.22$ & 14.22 \\
125 & $14.14 \pm 0.35$ & $13.12 \pm 0.38$ & $13.03 \pm 0.41$ & $14.39 \pm 0.20$ & 13.03 \\
130 & $12.96 \pm 0.32$ & $12.03 \pm 0.35$ & $12.01 \pm 0.37$ & $13.23 \pm 0.19$ & 11.97 \\
135 & $11.92 \pm 0.29$ & $11.07 \pm 0.33$ & $11.10 \pm 0.34$ & $12.20 \pm 0.17$ & 11.02 \\
140 & $10.99 \pm 0.27$ & $10.21 \pm 0.31$ & $10.29 \pm 0.32$ & $11.28 \pm 0.16$ & 10.18 \\
145 & $10.15 \pm 0.26$ & $9.44 \pm 0.29$ & $9.55 \pm 0.29$ & $10.45 \pm 0.15$ & 9.42 \\
150 & $9.40 \pm 0.24$ & $8.75 \pm 0.27$ & $8.89 \pm 0.27$ & $9.71 \pm 0.14$ & 8.74 \\
155 & $8.73 \pm 0.23$ & $8.13 \pm 0.25$ & $8.30 \pm 0.25$ & $9.04 \pm 0.14$ & 8.13 \\
160 & $8.12 \pm 0.21$ & $7.56 \pm 0.24$ & $7.75 \pm 0.24$ & $8.43 \pm 0.13$ & 7.57 \\
165 & $7.56 \pm 0.20$ & $7.05 \pm 0.23$ & $7.26 \pm 0.23$ & $7.88 \pm 0.12$ & 7.07 \\
170 & $7.06 \pm 0.19$ & $6.59 \pm 0.21$ & $6.82 \pm 0.21$ & $7.38 \pm 0.12$ & 6.62 \\
175 & $6.60 \pm 0.18$ & $6.17 \pm 0.20$ & $6.41 \pm 0.20$ & $6.92 \pm 0.11$ & 6.20 \\
180 & $6.19 \pm 0.17$ & $5.79 \pm 0.19$ & $6.04 \pm 0.19$ & $6.51 \pm 0.11$ & 5.83 \\
185 & $5.80 \pm 0.16$ & $5.43 \pm 0.18$ & $5.70 \pm 0.18$ & $6.13 \pm 0.10$ & 5.48 \\
190 & $5.46 \pm 0.15$ & $5.11 \pm 0.17$ & $5.39 \pm 0.18$ & $5.78 \pm 0.10$ & 5.16 \\
200 & $4.84 \pm 0.14$ & $4.55 \pm 0.16$ & $4.83 \pm 0.16$ & $5.16 \pm 0.09$ & 4.60 \\
220 & $3.88 \pm 0.12$ & $3.67 \pm 0.14$ & $3.96 \pm 0.14$ & $4.20 \pm 0.08$ & 3.73 \\
240 & $3.18 \pm 0.10$ & $3.02 \pm 0.12$ & $3.32 \pm 0.13$ & $3.49 \pm 0.07$ & 3.09 \\
260 & $2.66 \pm 0.09$ & $2.55 \pm 0.10$ & $2.84 \pm 0.12$ & $2.96 \pm 0.06$ & 2.61 \\
280 & $2.28 \pm 0.08$ & $2.19 \pm 0.09$ & $2.48 \pm 0.11$ & $2.58 \pm 0.06$ & 2.26 \\
300 & $2.00 \pm 0.08$ & $1.94 \pm 0.09$ & $2.23 \pm 0.11$ & $2.29 \pm 0.06$ & 2.00 \\
\hline
\end{tabular}

Table 4: NNLO predictions for the production cross sections $\sigma\left(p p \rightarrow H^{0}+X\right)[p b]$ at LHC for $\sqrt{S}=$ $7 \mathrm{TeV}$. The errors refer to the $\pm 1 \sigma$ pdf uncertainties.

In Table 3 the predictions for the NNLO Higgs boson production cross section for $p \bar{p}$ annihilation at $\sqrt{S}=1.96 \mathrm{TeV}$ are compared for the distributions $[8-12]^{7}$ for the mass range $100 \leq M_{H} \leq 200 \mathrm{GeV}$. Here the largest cross section differences are those between MSTW08 and ABKM09 which amount to $+22 \%$ at $M_{H}=100 \mathrm{GeV}$ and the MSTW08 prediction is $+39 \%$ higher than the one of ABM10 in the present exclusion region around $M_{H}=160 \mathrm{GeV}$. This difference corresponds to $5.6 \sigma$ in the pdf-error and is due to both the different gluon densities and $\alpha_{s}$ values having been obtained in both analyses, as discussed above. The impact of the

\footnotetext{
${ }^{7}$ See also [35].
} 
combined H1 and ZEUS data is seen by comparing the cross sections for ABM10 and ABKM09 : Larger cross sections are obtained at low masses $M_{H} \leq 140 \mathrm{GeV}$, while for higher masses the cross sections become smaller than the ones by ABKM09. The cross sections predicted by JR and MSTW08 are compatible within $1 \sigma$ and those of HERAPDF are about $1.5 \sigma$ larger than those of ABM10. From the above discussion of the gluon distribution and the different values of $\alpha_{s}\left(M_{Z}^{2}\right)$ involved, these quantitative relations can be understood to a large extent. To establish firm exclusion bounds, cf. [36], the observed variation in the predicted cross sections has to be taken into account as an uncertainty.

In Tables 4 and 5 we compare the different NNLO predictions for $p p$-scattering at the current LHC energy of $7 \mathrm{TeV}$ and the anticipated one of $8 \mathrm{TeV}$ for the next running period [37] for the mass range of $100 \leq M_{H} \leq 300 \mathrm{GeV}$. A quite similar pattern is obtained for both cms energies.

\begin{tabular}{|c|c|c|c|c|c|}
\hline$M_{H}(\mathrm{GeV})$ & ABM10 $[8]$ & ABKM09 $[9]$ & JR $[10]$ & MSTW08 $[11]$ & HERAPDF $[12]$ \\
\hline 100 & $28.81 \pm 0.65$ & $26.81 \pm 0.74$ & $25.66 \pm 0.91$ & $28.85 \pm 0.38$ & 26.38 \\
110 & $23.71 \pm 0.54$ & $22.04 \pm 0.61$ & $21.31 \pm 0.72$ & $23.83 \pm 0.32$ & 21.74 \\
115 & $21.62 \pm 0.49$ & $20.09 \pm 0.56$ & $19.53 \pm 0.65$ & $21.77 \pm 0.29$ & 19.85 \\
120 & $19.78 \pm 0.46$ & $18.38 \pm 0.51$ & $17.95 \pm 0.59$ & $19.96 \pm 0.27$ & 18.18 \\
125 & $18.15 \pm 0.42$ & $16.86 \pm 0.48$ & $16.55 \pm 0.53$ & $18.35 \pm 0.25$ & 16.70 \\
130 & $16.70 \pm 0.39$ & $15.52 \pm 0.44$ & $15.29 \pm 0.49$ & $16.93 \pm 0.23$ & 15.39 \\
135 & $15.41 \pm 0.36$ & $14.32 \pm 0.40$ & $14.17 \pm 0.44$ & $15.65 \pm 0.21$ & 14.21 \\
140 & $14.25 \pm 0.34$ & $13.24 \pm 0.38$ & $13.16 \pm 0.41$ & $14.51 \pm 0.20$ & 13.16 \\
145 & $13.21 \pm 0.32$ & $12.28 \pm 0.36$ & $12.26 \pm 0.37$ & $13.48 \pm 0.19$ & 12.22 \\
150 & $12.27 \pm 0.30$ & $11.41 \pm 0.33$ & $11.44 \pm 0.35$ & $12.55 \pm 0.18$ & 11.37 \\
155 & $11.42 \pm 0.28$ & $10.63 \pm 0.31$ & $10.69 \pm 0.32$ & $11.71 \pm 0.17$ & 10.60 \\
160 & $10.66 \pm 0.26$ & $9.92 \pm 0.29$ & $10.02 \pm 0.30$ & $10.96 \pm 0.16$ & 9.90 \\
165 & $9.96 \pm 0.25$ & $9.28 \pm 0.27$ & $9.41 \pm 0.28$ & $10.27 \pm 0.15$ & 9.27 \\
170 & $9.33 \pm 0.23$ & $8.69 \pm 0.26$ & $8.85 \pm 0.27$ & $9.64 \pm 0.14$ & 8.69 \\
175 & $8.75 \pm 0.22$ & $8.15 \pm 0.25$ & $8.34 \pm 0.25$ & $9.06 \pm 0.14$ & 8.17 \\
180 & $8.22 \pm 0.21$ & $7.67 \pm 0.24$ & $7.88 \pm 0.24$ & $8.54 \pm 0.13$ & 7.69 \\
185 & $7.73 \pm 0.20$ & $7.22 \pm 0.23$ & $7.45 \pm 0.23$ & $8.06 \pm 0.12$ & 7.25 \\
190 & $7.29 \pm 0.19$ & $6.81 \pm 0.21$ & $7.06 \pm 0.22$ & $7.62 \pm 0.12$ & 6.85 \\
200 & $6.51 \pm 0.18$ & $6.09 \pm 0.20$ & $6.36 \pm 0.20$ & $6.84 \pm 0.11$ & 6.14 \\
220 & $5.28 \pm 0.15$ & $4.96 \pm 0.17$ & $5.26 \pm 0.17$ & $5.61 \pm 0.10$ & 5.02 \\
240 & $4.37 \pm 0.13$ & $4.13 \pm 0.15$ & $4.44 \pm 0.15$ & $4.70 \pm 0.09$ & 4.19 \\
260 & $3.70 \pm 0.12$ & $3.51 \pm 0.13$ & $3.83 \pm 0.14$ & $4.03 \pm 0.08$ & 3.58 \\
280 & $3.20 \pm 0.10$ & $3.05 \pm 0.12$ & $3.38 \pm 0.14$ & $3.53 \pm 0.07$ & 3.13 \\
300 & $2.83 \pm 0.10$ & $2.72 \pm 0.11$ & $3.05 \pm 0.13$ & $3.17 \pm 0.07$ & 2.79 \\
\hline
\end{tabular}

Table 5: NNLO predictions for the production cross sections $\sigma\left(p p \rightarrow H^{0}+X\right)[p b]$ at LHC for $\sqrt{S}=$ $8 \mathrm{TeV}$. The errors refer to the $\pm 1 \sigma$ pdf uncertainties.

At $\sqrt{S}=7 \mathrm{TeV}$ the predictions of HERAPDF are lower than those of ABM10, while JR yields 
also smaller values for masses $M_{H}<200 \mathrm{GeV}$ and larger ones for higher Higgs masses. MSTW08 predicts higher cross sections than ABM10, with a tendency of a growing difference towards high masses. Overall the predictions are at variance of up to $3 \sigma$ of the pdf-errors, which corresponds to maximal deviations of 11-14\%. In the relevant region the gluon densities agree better than $5 \%$, but there is still also the uncertainty in $\alpha_{s}\left(M_{Z}^{2}\right)$, see Eq. (2). At $\sqrt{S}=8 \mathrm{TeV}$ the differences amount to less than $3.5 \sigma$ of the pdf-error, which corresponds to deviations of $11-16 \%$. In Table 6 we compare the NNLO predictions for $\sqrt{S}=14 \mathrm{TeV}$ for Higgs masses between 100 and $300 \mathrm{GeV}$. They differ between 10-14\% and agree better for larger masses. These differences form theory errors, which have to be accounted for within feasibility studies, cf. [38], and in searching for Higgs boson production at the LHC.

\begin{tabular}{|c|c|c|c|c|c|}
\hline$M_{H}(\mathrm{GeV})$ & ABM10 $[8]$ & ABKM09 $[9]$ & JR $[10]$ & MSTW08 $[11]$ & HERAPDF $[12]$ \\
\hline 100 & $71.16 \pm 1.53$ & $67.27 \pm 1.78$ & $62.24 \pm 2.62$ & $70.73 \pm 0.98$ & 65.54 \\
110 & $60.05 \pm 1.27$ & $56.60 \pm 1.48$ & $52.77 \pm 2.11$ & $59.73 \pm 0.81$ & 55.28 \\
115 & $55.42 \pm 1.17$ & $52.17 \pm 1.36$ & $48.82 \pm 1.92$ & $55.16 \pm 0.73$ & 51.01 \\
120 & $51.32 \pm 1.10$ & $48.25 \pm 1.24$ & $45.32 \pm 1.74$ & $51.10 \pm 0.69$ & 47.23 \\
125 & $47.63 \pm 1.00$ & $44.73 \pm 1.16$ & $42.16 \pm 1.59$ & $47.46 \pm 0.62$ & 43.83 \\
130 & $44.33 \pm 0.94$ & $41.59 \pm 1.08$ & $39.32 \pm 1.45$ & $44.19 \pm 0.57$ & 40.80 \\
135 & $41.36 \pm 0.87$ & $38.77 \pm 1.00$ & $36.77 \pm 1.33$ & $41.26 \pm 0.53$ & 38.07 \\
140 & $38.67 \pm 0.81$ & $36.22 \pm 0.93$ & $34.45 \pm 1.23$ & $38.60 \pm 0.49$ & 35.60 \\
145 & $36.23 \pm 0.77$ & $33.92 \pm 0.87$ & $32.36 \pm 1.13$ & $36.21 \pm 0.46$ & 33.37 \\
150 & $34.02 \pm 0.71$ & $31.83 \pm 0.81$ & $30.46 \pm 1.04$ & $34.03 \pm 0.43$ & 31.34 \\
155 & $32.00 \pm 0.67$ & $29.93 \pm 0.77$ & $28.72 \pm 0.97$ & $32.04 \pm 0.40$ & 29.49 \\
160 & $30.16 \pm 0.64$ & $28.20 \pm 0.72$ & $27.14 \pm 0.90$ & $30.22 \pm 0.38$ & 27.81 \\
165 & $28.48 \pm 0.62$ & $26.62 \pm 0.68$ & $25.70 \pm 0.83$ & $28.58 \pm 0.36$ & 26.28 \\
170 & $26.93 \pm 0.57$ & $25.16 \pm 0.65$ & $24.37 \pm 0.78$ & $27.05 \pm 0.34$ & 24.87 \\
175 & $25.52 \pm 0.54$ & $23.83 \pm 0.61$ & $23.15 \pm 0.73$ & $25.65 \pm 0.32$ & 23.58 \\
180 & $24.21 \pm 0.52$ & $22.61 \pm 0.58$ & $22.03 \pm 0.69$ & $24.37 \pm 0.31$ & 22.39 \\
185 & $23.00 \pm 0.49$ & $21.48 \pm 0.56$ & $20.99 \pm 0.64$ & $23.18 \pm 0.29$ & 21.30 \\
190 & $21.90 \pm 0.47$ & $20.44 \pm 0.53$ & $20.04 \pm 0.61$ & $22.09 \pm 0.28$ & 20.29 \\
200 & $19.91 \pm 0.43$ & $18.59 \pm 0.49$ & $18.33 \pm 0.55$ & $20.14 \pm 0.26$ & 18.49 \\
220 & $16.75 \pm 0.37$ & $15.64 \pm 0.41$ & $15.59 \pm 0.45$ & $17.03 \pm 0.22$ & 15.62 \\
240 & $14.38 \pm 0.33$ & $13.44 \pm 0.36$ & $13.54 \pm 0.38$ & $14.70 \pm 0.19$ & 13.46 \\
260 & $12.60 \pm 0.29$ & $11.79 \pm 0.33$ & $12.01 \pm 0.34$ & $12.96 \pm 0.18$ & 11.85 \\
280 & $11.27 \pm 0.27$ & $10.56 \pm 0.30$ & $10.86 \pm 0.30$ & $11.66 \pm 0.17$ & 10.64 \\
300 & $10.32 \pm 0.25$ & $9.69 \pm 0.28$ & $10.07 \pm 0.29$ & $10.75 \pm 0.16$ & 9.80 \\
\hline
\end{tabular}

Table 6: NNLO predictions for the production cross sections $\sigma\left(p p \rightarrow H^{0}+X\right)[p b]$ at LHC for $\sqrt{S}=$ $14 \mathrm{TeV}$. The errors refer to the $\pm 1 \sigma$ pdf uncertainties.

In Figure 3 we illustrate the different predictions of ABM10, JR, HERAPDF, and MSTW08 for the inclusive Higgs production cross section at the LHC for the energies $\sqrt{S}=7,10$ and 
$14 \mathrm{TeV}$. The cross sections rise roughly $\propto \sqrt{S}$. In this energy range ABM10 and MSTW08 predict nearly equal Higgs boson production cross sections, while those by JR and HERAPDF are found to be lower. For ABM10 and JR we include in the error also the scale variation uncertainty varying $\mu_{R}=\mu_{F}$ in the range $\left[M_{H} / 2,2 M_{H}\right]$ and added the pdf-errors in quadrature. It should be kept in mind that the scale variation errors are correlated when comparing the predictions for the different pdf sets. Although the various NNLO predictions differ w.r.t. the present pdf-errors, they are consistent within $1 \sigma$ taking the scale variation errors into account. At NNLO these are still significant, which will require to account for even higher order corrections, despite the well-known fact that soft gluon resummation leads to improvements (see [2] and references therein).

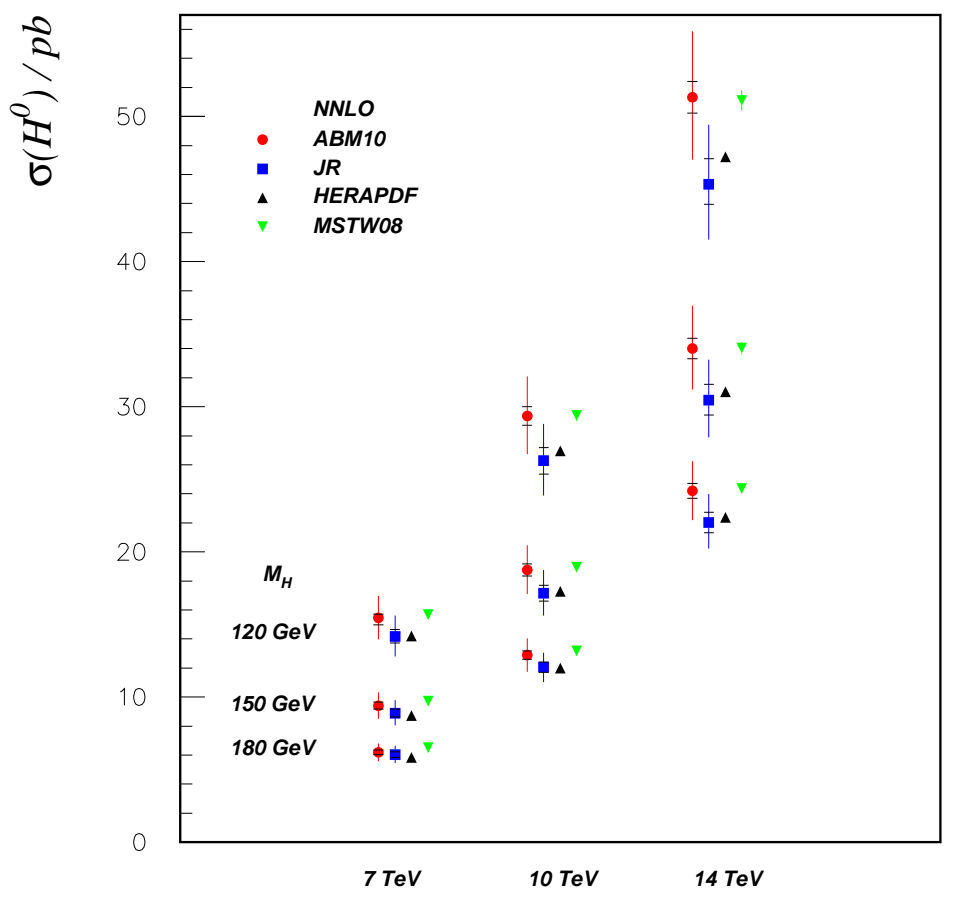

Figure 3: Predictions of the inclusive Higgs-boson production cross sections at NNLO for different energies at the LHC for the parton distributions ABM10, JR, HERAPDF, MSTW08, [8, 10-12]. For the ABM10 and JR distributions the scale variation errors corresponding to the range $M_{H} / 2 \leq \mu_{F}=$ $\mu_{R} \leq 2 M_{H}$ are included. The inner error bars refer to the pdf-errors only.

The typical scale uncertainty of each individual NNLO prediction amounts to about $\pm 9 \%$ at $7 \mathrm{TeV}$ and $\pm 8 \%$ at $14 \mathrm{TeV}$ which doubles at NLO [10,39]. A measure for the uncertainty of the Higgs boson production cross sections is thus obtained adding in quadrature the largest difference of central values and the largest error, including scale variations, of one of the predictions, and dividing this result by the central value of the smallest prediction. Higgs boson production at LHC can be predicted with an accuracy of about $10-17 \%$ at $\mathrm{NNLO}^{8}$ (with a total uncertainty being almost twice as large at NLO), whereas the uncertainty almost doubles at the Tevatron $(\sqrt{S}=1.96 \mathrm{TeV})$. Furthermore, the NNLO predictions are typically about $20 \%$ larger than at NLO.

We finally remark that a possible way to determine experimentally the gluon density in the mass region relevant for Higgs boson production consists in a precise measurement of the single

\footnotetext{
${ }^{8}$ Notice that the largest uncertainty refers to the predictions for $M_{H}=120 \mathrm{GeV}$ at $14 \mathrm{TeV}$, and the uncertainty decreases for $M_{H}$ increasing; the smallest uncertainty of about $10 \%$ refers to the predictions for $M_{H}=180 \mathrm{GeV}$ at $7 \mathrm{TeV}$.
} 
top quark production cross section [40-42] for scales around $\mu \sim m_{t}$. At present somewhat differing results for this process were obtained at Tevatron [43], however, still with large errors,

$$
\begin{aligned}
\sigma(p \bar{p} \rightarrow t(\bar{t})+\bar{b}(b)+X) & =2.3_{-0.5}^{+0.6} \text { (stat. + sys.) } p b \mathrm{CDF} \\
& =4.19_{-1.14}^{+1.24} \text { (stat. + sys.) } p b \text { D0 }
\end{aligned}
$$

with $^{9} V_{t b}=0.91 \pm 0.11$. Theoretical NLO QCD predictions ${ }^{10}$ are

$$
\begin{aligned}
\sigma(p \bar{p} \rightarrow t(\bar{t})+\bar{b}(b)+X) & =\left|V_{t b}\right|^{2}(2.420 \pm 0.003) p b & \text { MSTW08 } \\
& =\left|V_{t b}\right|^{2}(2.195 \pm 0.003) p b & \text { ABKM09 }
\end{aligned}
$$

which differ by about $10 \%$. The NLO scale variation errors according to $m_{t} / 2<\mu_{F, R}<2 m_{t}$ amount up to $10 \%$ at Tevatron, are lower than $5 \%$ at LHC [42], and can be further improved at NNLO. The different values in (5) and (6) are caused partly by yet different gluon distributions in the region $x \sim 0.1$, see Figure 2, but at least to the same extent they are due to the different values of $\alpha_{s}$, cf. Table 1. Precision measurements of the single top production cross sections at the LHC, although challenging, with an accuracy of $5 \%$ or better would allow to determine the gluon density in the same experiments which measure the production of the Higgs boson as well.

4 Conclusions. The present analysis shows that the current NNLO analyses of the world deep-inelastic data, supplemented with other relevant hard scattering data to measure the parton distributions, yield still different results at the level of less than about $10 \%$ for $W^{ \pm}$and $Z^{0}$ boson production, and at the level of $10-17 \%$ for Higgs boson production at the LHC. (These uncertainties would almost double at NLO.) The present variations in the predictions based on [8-13] are both due to the QCD-scale $\Lambda_{\mathrm{QCD}}\left(\right.$ or $\left.\alpha_{s}\left(M_{Z}^{2}\right)\right)$ and differences in the parton densties, as well as due to the renormalization and factorization scale uncertainties of the theoretical predictions. Future detailed work is needed to study and to further delineate these differences. The differences documented in this study form essential contributions to the theory error of the measurements of these scattering cross sections at the Tevatron and the LHC, which should therefore be compared to all the predictions based on the distributions ABM10 [8], JR [10], MSTW08 [11], and HERAPDF [12] at NNLO.

Acknowledgment. We would like to thank ECT* at Trento for hospitality and providing an inspiring environment at Villa Tambosi. For discussions we would like to thank S. Alioli, H. Böttcher, M. Cooper-Sarkar, G. Dissertori, A. Glazov, and M. Grazzini. This work was supported in part by DFG Sonderforschungsbereich Transregio 9, Computergestützte Theoretische Teilchenphysik, Helmholtz Alliance, the European Commission MRTN HEPTOOLS under the Contract No. MRTN-CT-2006-035505, and the Swiss National Science Foundation (SNF) under the Contract 200020-126691.

Appendix. In the following we summarize the different experimental measurements of the $W^{ \pm}$and $Z^{0}$-boson production cross sections in $p \bar{p}$ and $p p$ collisions for comparison with the predictions based on the current NNLO parton densities.

The cross sections for $p \bar{p}$ collisions measured by UA1, UA2 and D0 experiments are (averaging

\footnotetext{
${ }^{9}$ Assuming unitarity for the CKM matrix would imply a far smaller error [25].

${ }^{10}$ We would like to thank S. Alioli for computing the reference values for single top production with POWEG [41].
} 
over the different channels measured by UA1 for $Z^{0}$ production) :

$$
\begin{array}{rrl}
\text { UA1 }(0.546 \mathrm{TeV}): & \sigma^{W^{+}+W^{-}} B\left(W^{ \pm} \rightarrow \ell \nu\right) & =0.55 \pm 0.12 \quad n b \\
& \sigma^{Z^{0}} B\left(Z^{0} \rightarrow \ell^{+} \ell^{-}\right) & =0.070 \pm 0.046 \quad n b \\
\mathrm{UA2}(0.546 \mathrm{TeV}): & \sigma^{W^{+}+W^{-}} B\left(W^{ \pm} \rightarrow \ell \nu\right) & =0.57 \pm 0.08 \quad n b \\
& \sigma^{Z^{0}} B\left(Z^{0} \rightarrow \ell^{+} \ell^{-}\right) & =0.116 \pm 0.041 \quad n b \\
\mathrm{UA1}(0.630 \mathrm{TeV}): & \sigma^{W^{+}+W^{-}} B\left(W^{ \pm} \rightarrow \ell \nu\right) & =0.63 \pm 0.13 \quad n b \\
& \sigma^{Z^{0}} B\left(Z^{0} \rightarrow \ell^{+} \ell^{-}\right) & =0.070 \pm 0.019 \quad n b \\
\mathrm{UA2}(0.630 \mathrm{TeV}): & \sigma^{W^{+}+W^{-}} B\left(W^{ \pm} \rightarrow \ell \nu\right) & =0.57 \pm 0.08 \quad n b \\
& \sigma^{Z^{0}} B\left(Z^{0} \rightarrow \ell^{+} \ell^{-}\right) & =0.073 \pm 0.016 \quad n b \\
\text { D0(0.630 TeV }): & \sigma^{W^{+}+W^{-}} B\left(W^{ \pm} \rightarrow \ell \nu\right) & =0.658 \pm 0.058 \pm 0.034 \quad n b .
\end{array}
$$

The cross section measurement of CDF at $\sqrt{S}=0.630 \mathrm{TeV}$ has not been published [44].

At similar energies of $\sqrt{S}=0.5 \mathrm{TeV}$ the inclusive $W^{ \pm}$boson cross sections were recently measured by the PHENIX experiment [17] in $p p$ collisions at RHIC :

PHENIX $(0.5 \mathrm{TeV})$ :

$$
\begin{aligned}
& \sigma^{W^{+}} B\left(W^{+} \rightarrow e^{+} \nu\right)=0.1441 \pm 0.0212 \underset{-0.0103}{+0.0034} \pm 15 \% \text { (norm) } n b \\
& \sigma^{W^{-}} B\left(W^{-} \rightarrow e^{-} \bar{\nu}\right)=0.0317 \pm 0.0212 \underset{-0.0082}{+0.0101} \pm 15 \% \text { (norm) } n b .
\end{aligned}
$$

Here and in the following the errors are given in the sequence of statistical, systematic, and luminosity/normalization errors.

The $p \bar{p}$ experiments CDF and D0 at the Tevatron performed inclusive measurements for $W^{ \pm}$ and $Z^{0}$ production at $\sqrt{S}=1.8$ and $1.96 \mathrm{TeV}$ :

$\mathrm{CDF}(1.8 \mathrm{TeV})[18]$ :

$$
\begin{aligned}
\sigma^{W^{+}+W^{-}} B\left(W^{ \pm} \rightarrow \ell \nu\right) & =2.49 \pm 0.12 \quad n b \\
\sigma^{Z^{0}} B\left(Z^{0} \rightarrow \ell^{+} \ell^{-}\right) & =0.231 \pm 0.012 n b
\end{aligned}
$$

D0(1.8 TeV) [20] :

$$
\begin{aligned}
& \sigma^{W^{+}+W^{-}} B\left(W^{ \pm} \rightarrow \ell \nu\right)=2.310 \pm 0.001 \pm 0.005 \pm 0.100 \quad n b \\
& \sigma^{Z^{0}} B\left(Z^{0} \rightarrow \ell^{+} \ell^{-}\right)=0.221 \pm 0.003 \pm 0.004 \pm 0.010 n b
\end{aligned}
$$

$\operatorname{CDF}(1.96 \mathrm{TeV}) \quad[18,19]$ :

$$
\begin{aligned}
\sigma^{W^{+}+W^{-}} B\left(W^{ \pm} \rightarrow \ell \nu\right) & =2.749 \pm 0.010 \pm 0.053 \pm 0.165 \quad n b \\
\sigma^{Z^{0}} B\left(Z^{0} \rightarrow \ell^{+} \ell^{-}\right) & =0.2549 \pm 0.0033 \pm 0.0045 \pm 0.0152 \quad n b \\
& =0.2566 \pm 0.0007 \pm 0.0020 \pm 0.0154 \quad n b
\end{aligned}
$$


First measurements of the inclusive $W^{ \pm}$and $Z^{0}$ boson cross sections at LHC were performed at $\sqrt{S}=7 \mathrm{TeV}$ :

$$
\begin{array}{rlrl}
\text { CMS [6]: } \sigma^{W^{+}+W^{-}} B\left(W^{ \pm} \rightarrow \ell \nu\right) & =9.951 \pm 0.073 \pm 0.280 \pm 1.095 & n b \\
\sigma^{W^{+}} B\left(W^{+} \rightarrow \ell^{+} \nu\right) & =5.859 \pm 0.059 \pm 0.168 \pm 0.645 & n b \\
\sigma^{W^{-}} B\left(W^{-} \rightarrow \ell^{-} \bar{\nu}\right) & =4.140 \pm 0.064 \pm 0.254 \pm 0.455 & n b \\
\sigma^{Z^{0}} B\left(Z^{0} \rightarrow \ell^{+} \ell^{-}\right) & =0.960 \pm 0.037 \pm 0.059 \pm 0.106 & n b \\
\text { ATLAS [7]: } \sigma^{W^{+}+W^{-}} B\left(W^{ \pm} \rightarrow \ell \nu\right) & =9.96 \pm 0.23 \pm 0.50 \pm 1.10 & n b & \\
\sigma^{W^{+}} B\left(W^{+} \rightarrow \ell \nu\right) & =5.93 \pm 0.17 \pm 0.30 \pm 0.65 & n b & \\
\sigma^{W^{-}} B\left(W^{-} \rightarrow \ell^{-} \bar{\nu}\right) & =4.00 \pm 0.15 \pm 0.20 \pm 0.44 & n b \\
\sigma^{Z^{0}} B\left(Z^{0} \rightarrow \ell^{+} \ell^{-}\right) & =0.82 \pm 0.06 \pm 0.05 \pm 0.09 & n b .
\end{array}
$$

These cross sections correspond to analyzed samples of $2.88 \mathrm{pb}^{-1}$ and $0.32 \mathrm{pb}^{-1}$, while the current run may accumulate $O\left(50 \mathrm{pb}^{-1}\right)$, which will lead to a significant reduction of the statistical error and allow to improve the systematic errors.

To compare with the theoretical predictions one may use the current measured branching fractions $[25]$

$$
\begin{aligned}
& B\left(W^{+} \rightarrow e^{+} \nu_{e}\right)=0.1075 \pm 0.0013, \quad B\left(W^{+} \rightarrow \mu^{+} \nu_{\mu}\right)=0.1057 \pm 0.0015 \\
& B\left(W^{+} \rightarrow \tau^{+} \nu_{\tau}\right)=0.1125 \pm 0.0020
\end{aligned}
$$

with $B\left(W^{-} \rightarrow \ell^{-} \bar{\nu}_{l}\right)=B\left(W^{+} \rightarrow \ell^{+} \nu_{l}\right)$ and

$$
\begin{aligned}
& B\left(Z^{0} \rightarrow e^{+} e^{-}\right)=0.03363 \pm 0.00004, \quad B\left(Z^{0} \rightarrow \mu^{+} \mu^{-}\right)=0.03366 \pm 0.00007 \\
& B\left(Z^{0} \rightarrow \tau^{+} \tau^{-}\right)=0.03367 \pm 0.00008
\end{aligned}
$$

or their corresponding averages.

\section{References}

[1] T. Matsuura, S. C. van der Marck, W. L. van Neerven, Nucl. Phys. B319 (1989) 570;

R. Hamberg, W. L. van Neerven, T. Matsuura, Nucl. Phys. B359 (1991) 343;

V. Ravindran, J. Smith, W. L. van Neerven, Nucl. Phys. B682 (2004) 421 [hep$\mathrm{ph} / 0311304]$;

J. Blümlein and V. Ravindran, Nucl. Phys. B716 (2005) 128 [hep-ph/0501178];

R. Gavin, Y. Li, F. Petriello, S. Quackenbush, arXiv:1011.3540 [hep-ph].

[2] S. Catani, D. de Florian, M. Grazzini, JHEP 0105 (2001) 025 [hep-ph/0102227];

R. V. Harlander and W. B. Kilgore, Phys. Rev. D64 (2001) 013015 [hep-ph/0102241];

Phys. Rev. Lett. 88 (2002) 201801 [hep-ph/0201206];

C. Anastasiou, K. Melnikov, Nucl. Phys. B646 (2002) 220 [hep-ph/0207004];

V. Ravindran, J. Smith, W. L. van Neerven, Nucl. Phys. B665 (2003) 325 [hepph/0302135]; Nucl. Phys. B704 (2005) 332 [hep-ph/0408315];

S. Moch and A. Vogt, Phys. Lett. B631 (2005) 48 [hep-ph/0508265];

D. de Florian and M. Grazzini, Phys. Lett. B 674 (2009) 291 [arXiv:0901.2427 [hep-ph]];

R. V. Harlander and K. J. Ozeren, JHEP 0911, 088 (2009) [arXiv:0909.3420 [hep-ph]];

A. Pak, M. Rogal, M. Steinhauser, JHEP 1002 (2010) 025 [arXiv:0911.4662 [hep-ph]]. 
[3] For a survey, see U. Baur, Int. J. Mod. Phys. E17 (2008) 826 [hep-ph/0701164] and references therein.

[4] N. E. Adam, T. Aziz, J. R. Andersen et al., Higgs Working Group Summary Report, [arXiv:0803.1154 [hep-ph]] and references therein.

[5] C. Anastasiou, R. Boughezal, F. Petriello, JHEP 0904 (2009) 003 [arXiv:0811.3458 [hep$\mathrm{ph}]$.

[6] CMS collaboration, CMS PAS EWK-10-002 (2010); and in preparation: see talk by S. Stoynev at: 'Standard Model Benchmarks at the Tevatron and the LHC' Fermilab, Nov. 19-20, 2010, http://indico. cern. ch/conferenceDisplay.py?conf Id=101297.

[7] ATLAS collaboration, CERN-PH-EP 2010-037, arxiv:1010.2130 [hep-ex].

[8] S. Alekhin, J. Blümlein, S. Moch, arXiv:1007.3657 [hep-ph].

[9] S. Alekhin, J. Blümlein, S. Klein, S. Moch, Phys. Rev. D81 (2010) 014032 [arXiv:0908.2766 [hep-ph]].

[10] P. Jimenez-Delgado, E. Reya, Phys. Rev. D79 (2009) 074023 [arXiv:0810.4274 [hep-ph]]; Phys. Rev. D80 (2009) 114011 [arXiv:0909.1711 [hep-ph]].

[11] A. D. Martin, W. J. Stirling, R. S. Thorne, G. Watt, Eur. Phys. J. C63 (2009) 189 [arXiv:0901.0002 [hep-ph]].

[12] HERAPDF collaboration, H1-prelim-10-044, ZEUS-prel-10-008; https://www.desy.de/h1zeus/combined_results/proton_structure/Fits/HERAPDF1.0_NNLO_1145. LHgrid.gz

[13] A. D. Martin, W. J. Stirling, R. S. Thorne, G. Watt, arXiv:1007.2624 [hep-ph].

[14] M. Buza, Y. Matiounine, J. Smith, W. L. van Neerven, Eur. Phys. J. C 1 (1998) 301 [hep$\mathrm{ph} / 9612398]$;

I. Bierenbaum, J. Blümlein, S. Klein, Phys. Lett. B 672 (2009) 401 [arXiv:0901.0669 [hep$\mathrm{ph}]$.

[15] C. Albajar et al. [UA1 Collaboration], Z. Phys. C 44 (1989) 15.

[16] J. Alitti et al. [UA2 Collaboration], Z. Phys. C 47 (1990) 11.

[17] A. Adare, S. Afanasiev, C. Aidala et al., [PHENIX Collaboration], arXiv:1009.0505 [hep-ex]; see also: M. M. Aggarwal et al. [ STAR Collaboration ], arXiv:1009.0326 [hep-ex].

[18] A. Abulencia et al. [CDF Collaboration], J. Phys. G34 (2007) 2457 [hep-ex/0508029] and references therein.

[19] T. A. Aaltonen et al. [CDF Collaboration], Phys. Lett. B 692 (2010) 232 [arXiv:0908.3914 [hep-ex]].

[20] S. Abachi et al. [ D0 Collaboration ], Phys. Rev. Lett. 75 (1995) 1456 [hep-ex/9505013]; B. Abbott et al. [ D0 Collaboration ], Phys. Rev. D60 (1999) 052003 [hep-ex/9901040]; Phys. Rev. D61, 072001 (2000) [hep-ex/9906025]. 
[21] See e.g. talk by W.J. Stirling at the Workshop 'QCD at the LHC', ECT* Trento, Italy, Sept. 27 - Oct.1, 2010, http: //indico. cern. ch/conferenceOtherViews. py?view=standard\&conf Id=93790; PDF4LHC Interim Report, in preparation.

[22] G. Altarelli, QCD and experiment: Status of $\alpha_{s}$, CERN-TH-6623-92, in: Proceedings "QCD: 20 Years Later", Eds. P.M. Zerwas and H.A. Kastrup, (World Scientific, Singapore, 1993), pp. 172.

[23] J. Blümlein, S. Riemersma, W. L. van Neerven, A. Vogt, Nucl. Phys. Proc. Suppl. 51C (1996) 97 [hep-ph/9609217].

[24] F. D. Aaron et al. [ H1 and ZEUS Collaboration ], JHEP 1001 (2010) 109 [arXiv:0911.0884 [hep-ex]].

[25] N. Nakamura et al., (Particle Data Group), J. Phys. G37 (2010) 075021.

[26] P. M. Nadolsky et al., Phys. Rev. D 78 (2008) 013004 [arXiv:0802.0007 [hep-ph]].

[27] R. D. Ball, L. Del Debbio, S. Forte, A. Guffanti, J. I. Latorre, J. Rojo, M. Ubiali, Nucl. Phys. B 838 (2010) 136 [arXiv:1002.4407 [hep-ph]] and private communication.

[28] M. Glück, P. Jimenez-Delgado, E. Reya, C. Schuck, Phys. Lett. B 664 (2008) 133 [arXiv:0801.3618 [hep-ph]].

[29] A. D. Martin, W. J. Stirling, R. S. Thorne, G. Watt, Eur. Phys. J. C64 (2009) 653 [arXiv:0905.3531 [hep-ph]].

[30] P. Bolzoni, F. Maltoni, S. Moch, M. Zaro, Phys. Rev. Lett. $105 \quad$ (2010) 011801. [arXiv:1003.4451 [hep-ph]].

[31] J. Blümlein, H. Böttcher, A. Guffanti, Nucl. Phys. B774 (2007) 182 [hep-ph/0607200]; Nucl. Phys. Proc. Suppl. 135 (2004) 152 [hep-ph/0407089].

[32] J. Blümlein and H. Böttcher, Nucl. Phys. B841 (2010) 205 [arXiv:1005.3113 [hep-ph]].

[33] S. Bethke, Eur. Phys. J. C64 (2009) 689 [arXiv:0908.1135 [hep-ph]].

[34] T. Gehrmann, M. Jaquier, G. Luisoni, Eur. Phys. J. C67 (2010) 57 [arXiv:0911.2422 [hep$\mathrm{ph}]$;

R. Abbate, M. Fickinger, A. H. Hoang, V. Mateu, I.W. Stewart, [arXiv:1006.3080 [hep-ph]];

R. Frederix, S. Frixione, K. Melnikov, G. Zanderighi, arXiv:1008.5313 [hep-ph].

[35] J. Baglio and A. Djouadi, JHEP 1010, 064 (2010) [arXiv:1003.4266 [hep-ph]];

V. Ahrens, T. Becher, M. Neubert, L. L. Yang, arXiv:1008.3162 [hep-ph].

[36] TEVNPH Working Group [CDF and D0 Collaborations], arXiv:1007.4587 [hep-ex].

[37] R.D. Heuer, private communication.

[38] ATLAS Collaboration, ATL-PHYS-PUB-2010-15.

[39] R. Harlander, J. Phys. G35 (2008) 033001. 
[40] J. M. Campbell, R. K. Ellis, F. Tramontano, Phys. Rev. D70 (2004) 094012 [hepph/0408158];

S. Frixione, E. Laenen, P. Motylinski, B.R. Webber, JHEP 0603 (2006) 092 [hep$\mathrm{ph} / 0512250]$;

J. M. Campbell, R. Frederix, F. Maltoni, F. Tramontano, JHEP 0910 (2009) 042 [arXiv:0907.3933 [hep-ph]];

[41] S. Alioli, P. Nason, C. Oleari, E. Re, JHEP 0909 (2009) 111 [arXiv:0907.4076 [hep-ph]].

[42] J. M. Campbell, R. Frederix, F. Maltoni, F. Tramontano, Phys. Rev. Lett. 102 (2009) 182003 [arXiv:0903.0005 [hep-ph]].

[43] T. Aaltonen et al. [ CDF Collaboration ], Phys. Rev. Lett. 103 (2009) 092002 [arXiv:0903.0885 [hep-ex]]; arXiv:1004.1181 [hep-ex]; V. M. Abazov et al. [ D0 Collaboration ], Phys. Lett. B682 (2010) 363 [arXiv:0907.4259 [hep-ex]].

[44] E. James, private communication, Oct. 202010. 